UC-4

issued: November 1976

\title{
Uranium Daughter Growth Must Not Be Neglected When Adjusting Plutonium \\ Materials for Assay and Isotopic Contents
}

by

S. F. Marsh

W. D. Spall

R. M. Abernathey

J. E. Rein

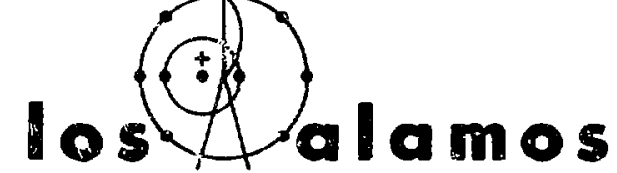

scientific laboratory

of the University of California

LOS ALAMOS, NEW MEXICO 87545

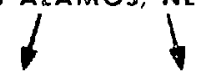

An Alfirmative Action / Equal Opportunity Employer 
This work supported by the US Energy Research and Develupment Administration, Division of Safeguards and Security and Division of Reactor Develepment and Demonstration.

Printed in the United States of America. Available from

National Technical Information Service

U.S. Department of Commerce

5285 Port Royal Road

Springfield, VA 22161

Price: Printed Copy $\$ 3.50$ Microfiche $\$ 3.00$

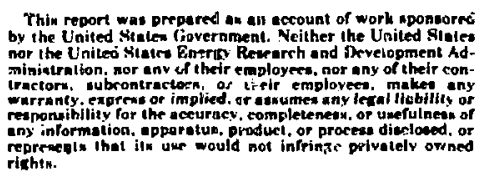

reprewe 


\title{
6RANIUM DAUGHTER GROW'TH MUST NOT BE NEGLECTED WHEN ADJUSTING PLUTONIUM MATERIALS FOR ASSAY ANE ISOTOPIC CONTENTS
}

\author{
by
}

\author{
S. F. Marsh, W. D. Spall,
}

T. M. Abernathey, and J. E. Rein

\begin{abstract}
ARSTEACT
Relationships are provided to compute the decreasing plutonium content and changing isotopic distribution of plutonium materials for the radioactive decay of ${ }^{236} \mathrm{Pu},{ }^{230} \mathrm{Pu},{ }^{200} \mathrm{Pu}$, and ${ }^{242} \mathrm{Pu}$ to long-lived uranium daughters and of ${ }^{111} \mathrm{Pu}$ to ${ }^{201} \mathrm{Am}$. This computation is important to the use of plutonium reference materials to calibrate destructive and nondestructive methods for assay and isotopic measurements, as well as to accountability inventory calculations.
\end{abstract}

\section{INTRODUCTION}

Calculation of the decreasing plutonium content and changing isotopic distribution of plutonium reference materials fo- the radioactive decay of ${ }^{241} \mathrm{Pu}$ to ${ }^{341} \mathrm{Am}$ is generally applied in analytical chemistry laboratories. Not usually recognized is that the effects of the radioactive decay of ${ }^{200} \mathrm{Pu},{ }^{200} \mathrm{Pu},{ }^{200} \mathrm{Pu}$, and ${ }^{902} \mathrm{Pu}$ can exceed that of ${ }^{241} \mathrm{Pu}$ decay, depending on the plutoinium isotopic distribution of the material. This is the case especially for materials having low ${ }^{24} \mathrm{Pu}$ isotopic abundances, a characteristic of presently available National Bureau of Standards "Standard Reference Materials" (SRM) certified for plutonium assay use.

The calculational relationships in this report provide, as a function of time, (a) the plutonium content, (b) grown-ir quantities of ${ }^{284} \mathrm{U},{ }^{280} \mathrm{U},{ }^{380} \mathrm{U}$, ${ }^{888} \mathrm{U}$, and ${ }^{24} \mathrm{Am},(\mathrm{c})$ plutonium isotopic distribution, and (d) plutonium atomic weight. The Fortran IV program for these calculations is provided as an appendix.

\section{DISCUSSION}

\section{A. G̈eneral}

This repor was perice This report was prepared at an account of woik

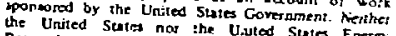

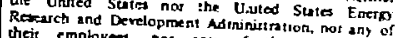
theis emploves. nos say of ther contractors subcontractory. of their cmployces, mates an mirtanty. express os implues, of esturnes any tegal bibility or responstbitty for the acciuracy, sompletexes or uxfulnes of any informa tic appeisius, piletenes proces doctosed. On represents that its use would not

For a time interval $\Delta t$, th number if parent atoms decayed is

$N_{0}-N=N_{0}\left[1-e^{-(\Delta t)(\ln 2) /\left(t \frac{1}{2}\right)}\right]$. 
The published t values ${ }^{2,2}$ for the five plutonium isotopes given in Table I should be replaced by more reliable values as they become available. Table I also provides the atomic weights of the five plutonium isotcpes, four uranium daughter isotopes, and ${ }^{241} \mathrm{Am}$. Alpha decay, the main decay mode of ${ }^{20} \mathrm{Pu},{ }^{200} \mathrm{Pu},{ }^{240} \mathrm{Pu}$, and ${ }^{242} \mathrm{Pu}$, produces long-lived uranium daughters having essentially four fewer atomic mass units than the plutonium parente. The calculations account for these mass changes. Beta decay of ${ }^{21} \mathrm{Pu}$ to ${ }^{2 *} \mathrm{Am}$ does not change mass significantly, but coes, of course, change the plutonium content.

TABLE I

\section{PI.UTONIUM ISOTOPE HALF-LIVES AND ATOMIC WEIGHTS}

$\begin{array}{cccc}\text { Isotope } & \text { Half-Life, } \mathbf{Y} \mathbf{r}^{*} & & \text { Atomic Weight } \\ & & & \\ 238 & 87.8 & & 238.0495 \\ 239 & 24390 & & 239.0522 \\ 240 & 6540 & & 240.0538 \\ 241 & 14.7 & & 241.0569 \\ 242 & 387000 & & 242.0588\end{array}$

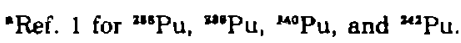

Pef. 2 for ${ }^{m} \mathrm{Pu}$.

\section{LFANIUM DAUGHTER AND ${ }^{211}$ AM ATOMIC WEIGHTS}

$\begin{array}{lr}\text { Isotope } & \text { Atomic Wei } \\ & \\ { }^{2860} \mathrm{~J} & 234.0410 \\ { }^{280} \mathrm{U} & 235.0439 \\ { }^{280} \mathrm{U} & 236.0456 \\ { }^{280} \mathrm{U} & 238.0508 \\ { }^{241} \mathrm{Am} & 241.0568\end{array}$

\section{B. Plutonium Mass Decrease}

The general equation giving the mass decrease of an individual plutonium isotope with time is

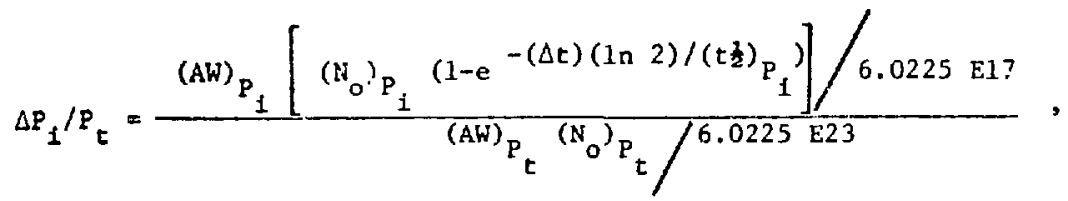

which reduces to

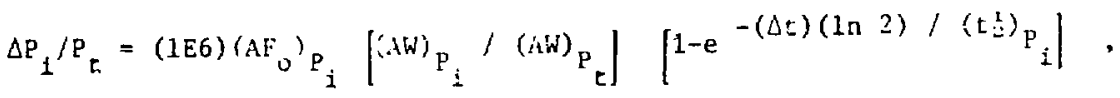


in which

$$
\begin{aligned}
& \Delta P_{1}=\text { micrograms decrease of individual plutonium isotopa, } P_{1} \\
& P_{t}=\text { grams total plutonium } \\
& (A W)_{P_{i}}=\text { atomic weight of } P_{1} \\
& (A W)_{P_{t}}=\text { atomic weight of } I^{\prime} \\
& \left(N_{0}\right)_{P_{i}}=\text { atoms of } P_{1} \text { per gram of } P_{t} \text { at initial time } \\
& \left(N_{0}\right)_{P_{r}}=\text { atoms of } P_{t} \text { per gram of } P_{t} \text { at initial time } \\
& \Delta t \quad=\text { decay time, years } \\
& (t)_{P_{i}}=\text { half-life, years, of } P_{1} \\
& \left(A F_{0}\right)_{P_{i}}=\text { atom abundance fraction of } P_{1} \text { at initial time. }
\end{aligned}
$$

The total mass decrease of plutonium is the sum of the five iridividual plutaniuns isotope mass decreases.

\section{Uranium Masa Increase}

The general equation giving the mass increase of a uranium daughter with time is

$$
\Delta \mathrm{U}_{1} / \mathrm{P}_{\mathrm{E}}=\left(\Delta \mathrm{P}_{1} / \mathrm{P}_{\mathrm{t}}\right) \quad\left[(\mathrm{AW})_{\mathrm{U}_{1}} /(\mathrm{AW})_{\mathrm{P}_{1}}\right]
$$

in which

$\Delta \mathrm{U}_{1}=$ micrograms increase of uranium daughter from decay of parent $P_{1}$

$(A W)_{v_{i}}=$ atomic weight of $U_{1}$.

The total mass increase of uranium is the sum of the four individual uranium isotope mass increases.

\section{D. ${ }^{201}$ Am Mass Increase}

The ${ }^{{ }^{11}} \mathrm{Am}$ mass increase is not significantly different from the ${ }^{201} \mathrm{Pu}$ mass decrease given by Eq. (3)

\section{E. Plutonium :}

The equation giving the decreasing plutonium content with time is

$$
A=\frac{A_{0}-A_{0} D / 1 E 6}{A_{0}-A_{0} D / 1 E 6+\left(1-A_{0}\right)+A_{0} G / 1 E 6} \text {, }
$$

which reduces to

$$
A=\frac{\left.A_{0}(1 E 6)-D\right)}{1 E 6-A_{0}(D-G)} \text {, }
$$


in which

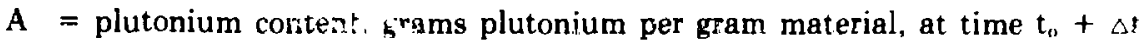

$A_{0}=$ plutonium content, $y r \varepsilon x$ :s plutonium per gram material, at time $t_{\mathrm{v}}$

D = total plutonium mass riscrease, micrograms plutcnium per gram initial plutonium, during time $\Delta t$

$\mathrm{G}=$ mass increase of ${ }^{2+1} \mathrm{Am}$ and total uranium, micrograms per gram initial plutonium, in time $\Delta \mathrm{t}$.

\section{F. Plutonium Isotopic Distribstion}

The basic equation applies:

$N_{P_{i}}=N_{{ }_{0} P_{i}}\left[e^{-(\Delta t)(\ln 2) /\left(t \frac{1}{2}\right)}\right]$.

The five $\mathrm{N}_{1}$ values are computed and renornialized to a total atom fraction of 1 (totai atom percentage of 100).

\section{G. Plutonium Atomic Weight}

The atomic weight of the plutonium at time $t_{0}+\Delta t$ is given by

${ }^{(A W)_{P_{t}}}=\sum\left[(A E)_{P_{i}} \quad{ }^{(A W)_{P_{i}}}\right]$,

in which

$(A W)_{P_{t}}=$ atomic weight of plutonium. at time $t_{0}+\Delta t$

$(\mathrm{AF})_{\mathrm{p}_{\mathrm{i}}}=$ renormalized atom fraction of individual p!utonium isotope calculated by use of $\mathrm{Eq}$. (7)

$(A W)_{P_{i}}=$ atomic weight of $P_{1}$.

\section{I11. EXAMPLE CALCULATION}

Table II gives the decreased plutonium content; grown-in quantities of ${ }^{284} \mathrm{U},{ }^{286} \mathrm{U},{ }^{280} \mathrm{U},{ }^{288} \mathrm{U}$, and ${ }^{241} \mathrm{Am}$; and changed plutonium isotopic distribution for a glutonium metal typical of a Nationai Bureau of Standards SRM at a 10-yr $\Delta$ t. For purposes of demonstration, the initial plutonium concent is taken as $1 \mathrm{~g}$ Pu per gram material (or $100 \%$ purity). It is noted that the plutonium content decreases $0.05 \%$ from $100.00 \%$ to $99.95 \%$ and that $0.02 \%$ (or 40 relative per cent of the $0.05 \%$ decrease) is caused by ${ }^{2 * 1} \mathrm{Pu}$ decay. 
TABLE II

EXAMPLE CALCULATION FOR 10-YR DECAY

\begin{tabular}{|c|c|c|c|c|c|}
\hline \multirow{2}{*}{$\begin{array}{c}\text { Pu } \\
\text { Isotope }\end{array}$} & \multirow[b]{2}{*}{$\left(\mathbf{A F _ { 0 }}\right)_{\mathbf{F}_{i}}$} & \multirow[b]{2}{*}{$(\mathbf{A F})_{P_{1}}$} & \multirow{2}{*}{$\begin{array}{c}\Delta \mathbf{P}_{1} / \mathbf{P}_{\mathrm{I}} \\
\boldsymbol{\mu \mathrm { B } / \mathbf { G }}\end{array}$} & \multicolumn{2}{|c|}{ Daughter Formed } \\
\hline & & & & Isotope & $\mu \mathrm{g} / \mathrm{g}$ \\
\hline 238 & 0.00003 & $0.00002 \%$ & 2.268 & ${ }^{284} U$ & 2.229 \\
\hline 239 & 0.97617 & 0.97638 & 277.353 & ${ }^{28} \mathrm{U}$ & 272.703 \\
\hline 240 & 0.02324 & 0.02322 & 24.719 & ${ }^{280} \mathrm{U}$ & 24.306 \\
\hline 241 & 0.00054 & 0.00033 & 204.693 & ${ }^{201} \mathrm{Am}$ & 204.693 \\
\hline 242 & 0.00002 & 0.000002 & 0.000 & ${ }^{28 B} \mathrm{U}$ & 0.000 \\
\hline$(A W)_{P}$ & 239.076 & & $\Sigma=509.033$ & & $\Sigma=503.931$ \\
\hline
\end{tabular}

\section{Plutonium Content}

$$
\begin{aligned}
A & =\frac{A_{0}(1 E 6-D)}{1 E 6-A_{0}(D-G)}=\frac{1(1 E 6-509.033)}{1 E 6-1(509.033-503.931)} \\
& \left.=0.99949_{6} \text { (or } 99.95 \%\right)
\end{aligned}
$$

Plutonium Atomic Weight

$$
\begin{aligned}
(A W)_{P_{t}}= & \sum\left|{ }^{(A F)_{P_{i}}}{ }^{\left(A W{ }_{P_{i}}\right.}\right| \\
= & \left(0.000027_{7}\right)(238.0495)+(0.97638)(239.0522)+\left(0.02322_{7}\right)(240.0538)+\left(0.00033_{7}\right) \\
& (241.0569)+\left(0.00002_{0}\right)(242.0588) \\
= & 239.0759 .
\end{aligned}
$$

\section{REFERENCES}

1. N. E. Holden and F. W. Walker, Chart of the Nuclides, Eleventh Edition, Knolls Atomic Power Laboratory (1972).

2. E. Garner, National Bureau of Standards, Personal Communication, April 1976. Also value recommender on National Bureai of Standards certificates for plutonium SRM. 


\title{
APPENDIX
}

\section{FORT:AN IF COMPUTATIONAL PROGRAM LASL IDENTIFICATION: LP'-0710}

\author{
ULI 10 PREGFFII FUILFY : IPIPUT - DUTPUT:

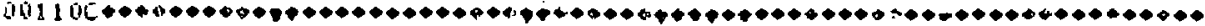 \\ 001300

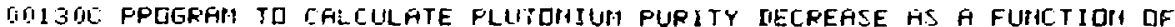 \\ 001400 TIHE. WFITTEH 5-12-76 FY 1, I IALE SPFL: FOF USE DH THE LASL 6600 \\ OOISOC RROIOS TIIIE SHARING SYSTEM. \\ 001600

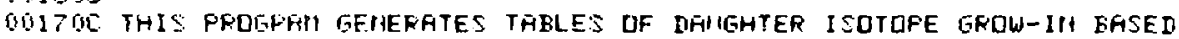

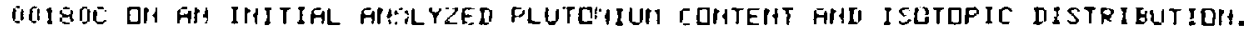

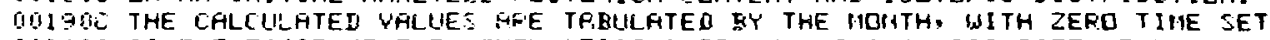 \\ WUEUO: AS THE FIRST DF THE MOISTH LIESIGHATED BY THE ARIHLYSIS DATE. THE \\ DIZIOI: VALUES CALLULATED GRE FUR DHE HDITH IMTEPVALS FFOI THAT DATE. CARE \\ OMZZUL MUST BE TAHEH III SELECTIHG THF STARTIPG MOITH UATE TD MIHIMIZE THE

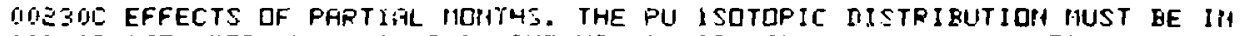

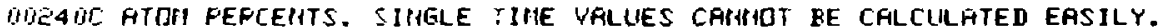 \\ 00250 \\ OOEG UIC VARIAFLE LIST

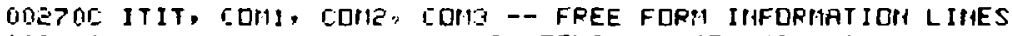

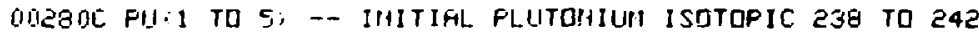

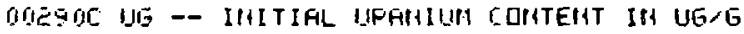 \\ 003000 EHH - - IHITIAL FIIEFICIUH COHTEITT IHA UG/G \\ 00100 PLII -- IMITIAL FLUTOHIUM PURITY IH PERCERT

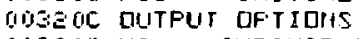

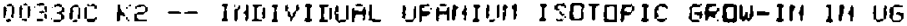

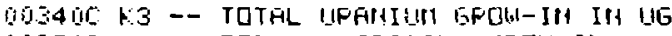

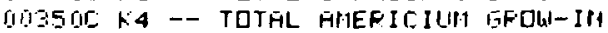

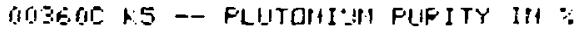

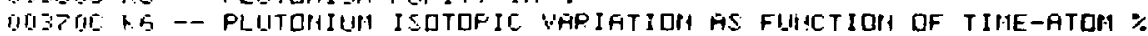 \\ UOSBOC: $Y$ - HIIIIEER OF YEHES TO FE CDUEFEL IH THE TAELES

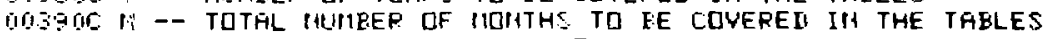

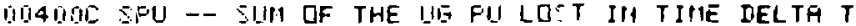 \\ DU4!IC SU - SUR DF IIG II GAIIEI III TIIIE IIELTA T

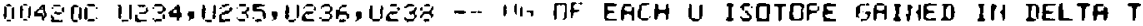

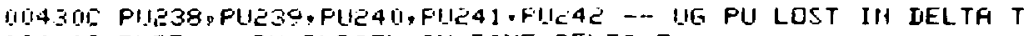

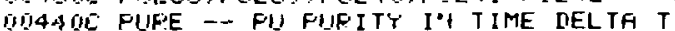

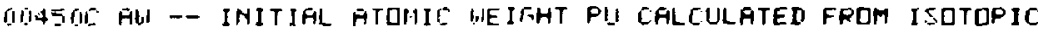

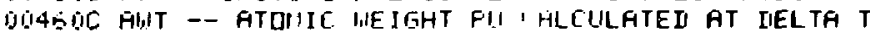

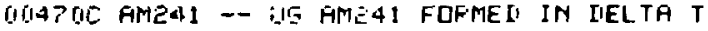

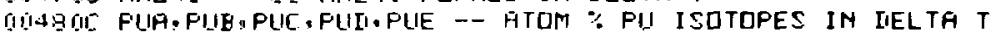 \\ DII 49 IIL TIM - IIELTA TIME IM IAECIMFL MONTHS

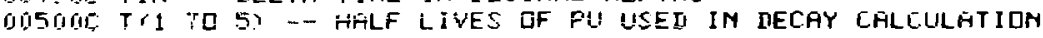 \\ $0.15,100$

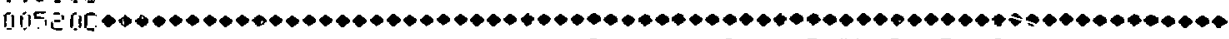

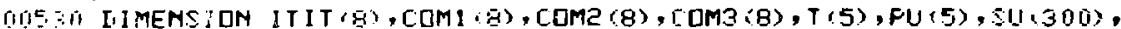

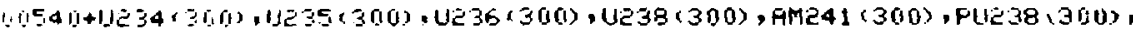

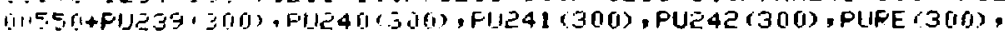

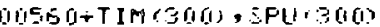 \\ 00570101 FFINT 100

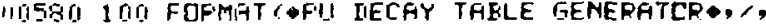

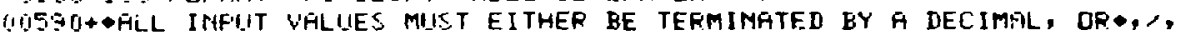

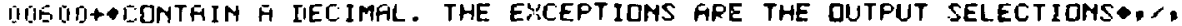

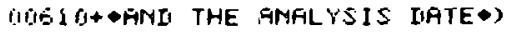 \\ IOSEO FFINT 110

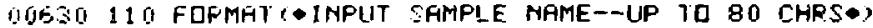 \\ $0064(1$ PEAI 120, (ITIT (I), I=1, \\ OUDSO 120 FDFMAT IBA:O \\ $006 S 0$ FFINT 130 \\ UIESU 1:0 FDFMAT ( INPUT LIP TO 3 LINES DF COINENTS DR 3 SPACE-EAR RETURNS $\bullet$ \\ 00680 REFI $12(1, \operatorname{COM} 1$ ( $I), I=1,8)$

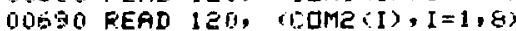 \\ TOPOO READ $12(1$, (COM3 (1), I=1,8) \\ OIII 1 PFINT 140
}


U1:-20 140 FOFMFTC IMPUT DFTE ANRLYZED HS MMYY

UUT 30 PEAL 150 , IIMTE, YERP.

GITAO 150 FQFPIATCZF2.OY

(i)

DIS6O 1SO FDFMATC INPUT PU ISOTOPIC IISTRIBUTION AS ATOM PEPCEMTS*, , DUESB*;

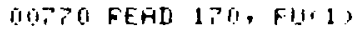

$0 B T E O$ EEO FQFMAT (II)

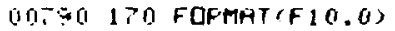

$00 O Q O$ FRINT 150

OUBID 190 FOFMAT

OIFZO PEAD 170 . FUL 2 )

00830 FFINT 190

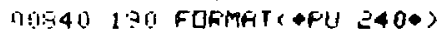

OUES0 FEAI 170 , Fil: 3 )

DOSBO FFIMT EOR:

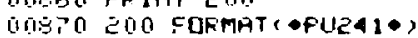

DIIBSO FEAI 170 ,PU(4)

(1ISB) FEINT 310

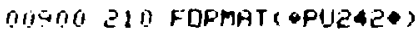

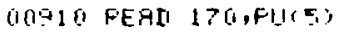

G0OZO PFINT CEO

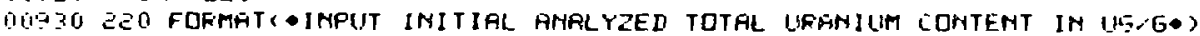

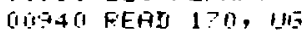

00,50 PPINT 230

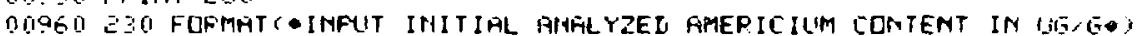

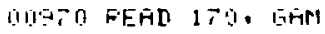

10.0\% PPINT 240

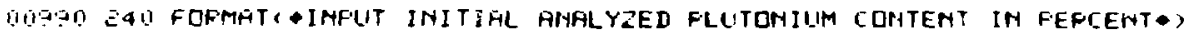

U1 DOUO FEAL 170 , FlII

(1)

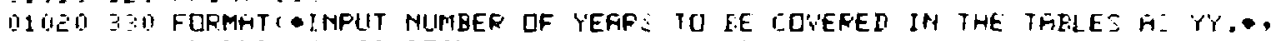

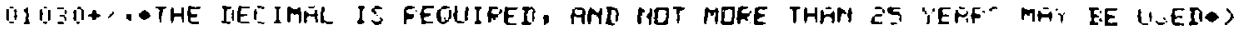

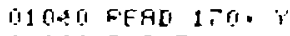

01050 PFINT 40

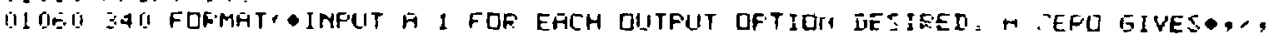

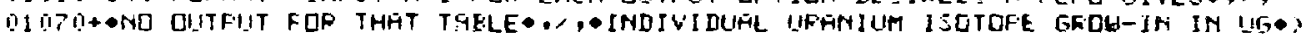

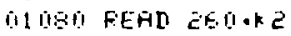

G1070 FFINT 350

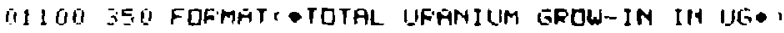

G1110 FEAI E.G.1.

011 OI FPINT OA

11130 SEO FOFMHT - TOTAL RMEFICILM GFOW-IN IN L15.'

II 14 S FEAI EE 14

(1) 150 FPINT $\because 1$

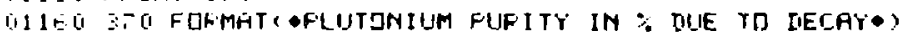

i.1 1 CO FEAD 260,15

(1) 1 SO PFINT 380

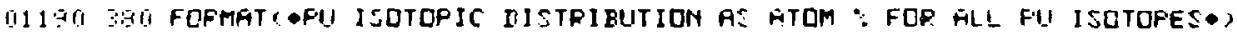

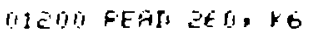

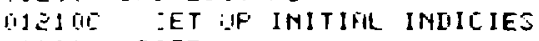

(1)EEO $r=\operatorname{TIFTE}$

$01 \geq 30 l=k+1$

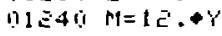

$0 ! E 50 \quad m=k+r_{1}$

T1EGOIL ZEFO INITIAL INE IGHT RFFFYS

ज1Ëत on 30 $\mathrm{I}=1$,

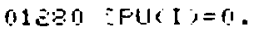

01 290 (1) I $3-0$.

01300 ण $34(1)=r$.

(1) 310 प $1235(1)=0$.

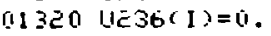

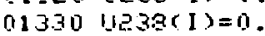

01340 Flle38 $(1)=0$.

01350 PUESS(I) $=0$.

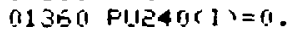

01370 FIIEA1 1 . $=0$. 


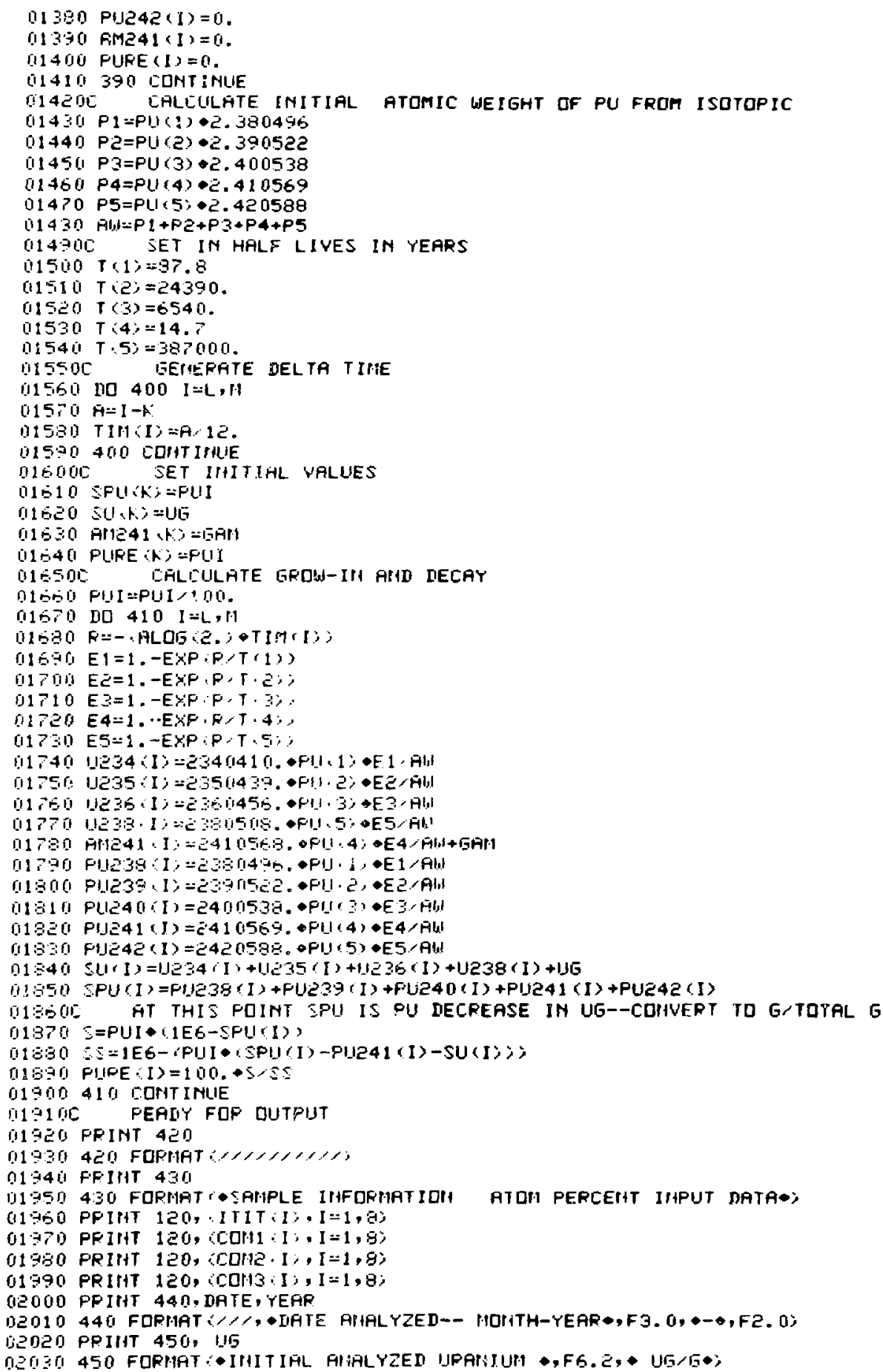




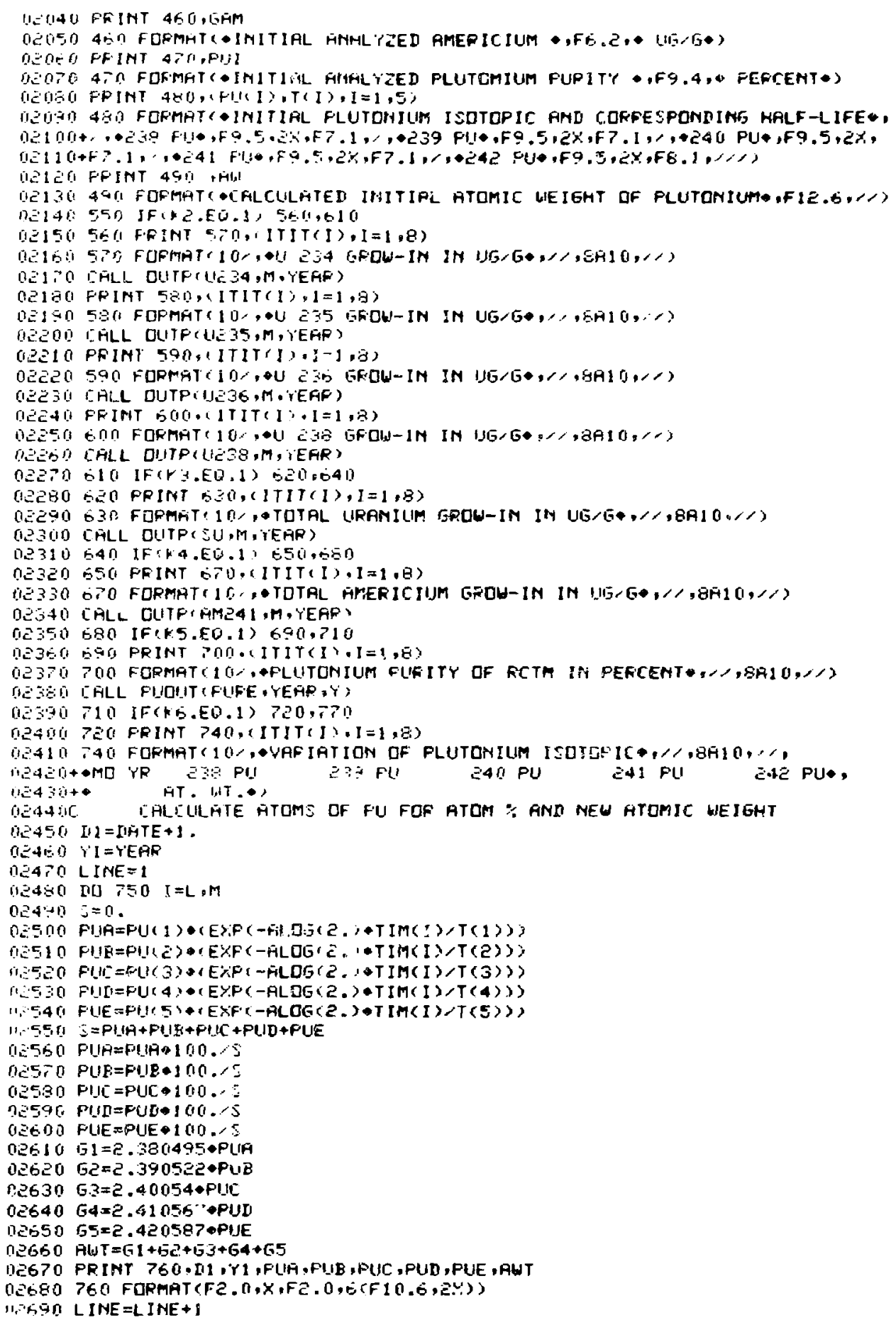




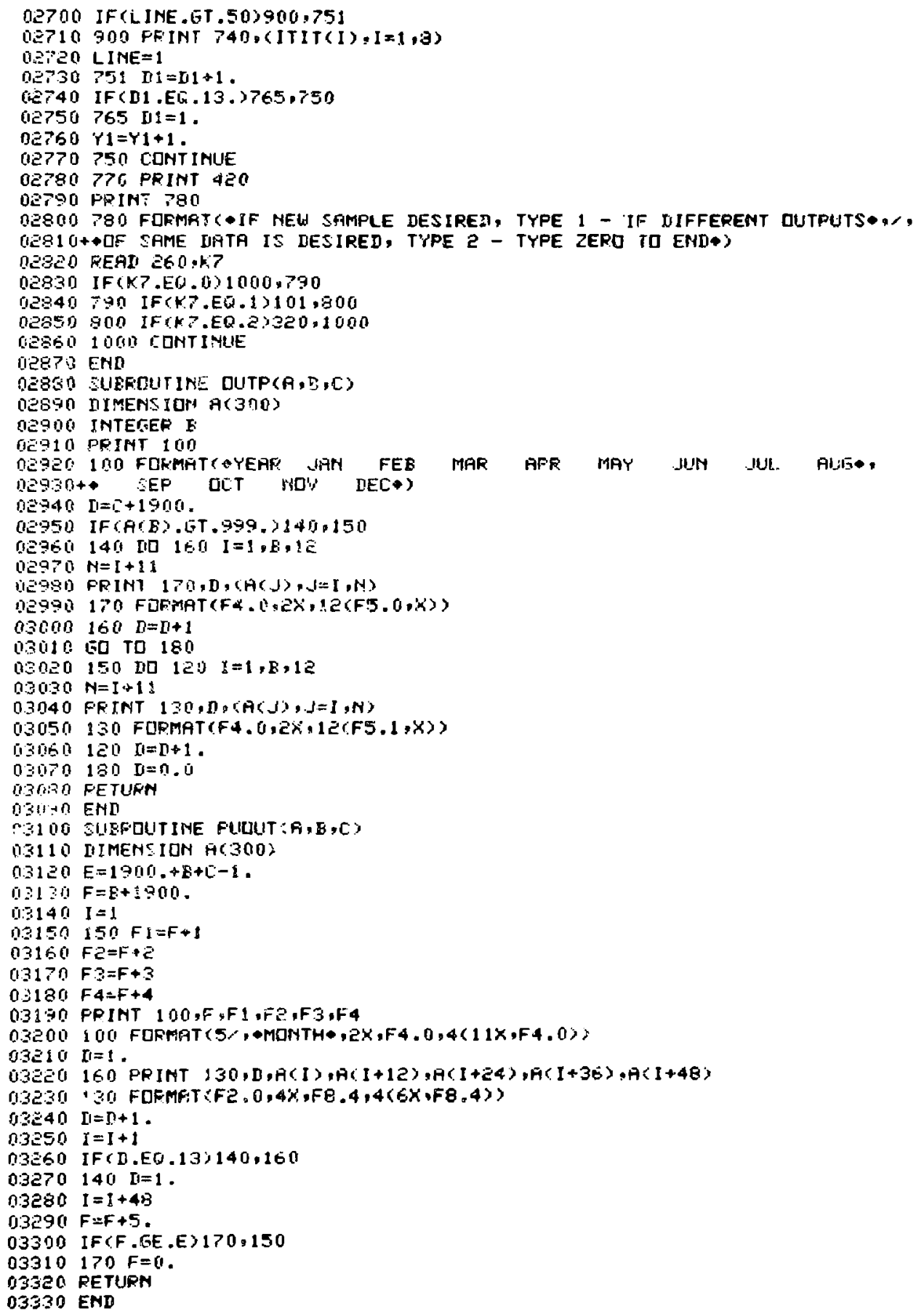


S.MHPLE IHFORMATIDII ATMH PERCEIT IIIPUT DATA

EXOMPLE LRLCULATIOH FOR IO YEAR DECAY PERIDD

THIS IS FRIPLY TYPICAL UF PLUTOHIUH METAL HATEPIRLS

THE I:IITIAL FUPITY HAS EEEIH SET TO $100 \%$ FDP THE SANE DF CLRRIT'Y

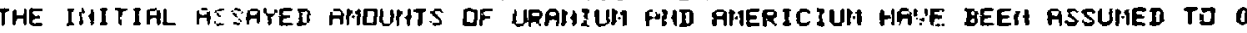

SATE AIIALYZED- - MDHTH-YEAR 1-76

IIII IAL APHPL YZED IFATIIUH 0 . UIG/G

IIII TIAL FIIHL YZED FHIEPICIUH O. UG/G

INITIAL FIALYZED PLUTUHIUN PURITY 1.0000 fERCENT

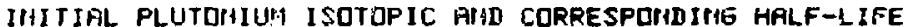

$238 \mathrm{PL} .00300 \quad 87.8$

239 PU $97.61780 \quad 24390.0$

240 PU 2.32400 6540.0

24: PU .05400 14.7

242 PU $.00200 \quad 387000.0$

CP!_CUL.ATED IHITIAL ATDMIC WEIGHT DF PLUTBHIUM 239.076590 
PU DECAY TABLE GEIERATDR

FLL IIPUT VALUES MUST EITHER BE TERMIPAATED BY A DECIMPL, DR COHTAIH A DECIHAL. THE EXCEPTIOHS ARE THE DUTPUT SELECTIOIS

AITD THE FIIFLYSIS DATE

IIIPUT SAPIPLE TYAPIE--IJF TO 80 CHRS

$?$ EXPMPI.E GRLCULATIOIS FOR 10 'Y'EAR DECAY PERIOD

IHPUT UP TC 3 LIHIES CF COHIEIITS OR 3 SPACE-GRR RETURHS

$?$ THIS IS FAIFLY TYPICHL DF FLUTDPIIIP HETAL MATERIPLS

? THE IIIITIAL PURITY HAS BEEI SET TD IOU\% FDR THE SAKE DF CLARITY

$?$ THE IHITIAL ASSAYED AMDUHTS OF URATILIM AIID AHERICIUH MAVE BEEII ASSUHED TD 0 IIPUT DATE FITALYZED AS MIYYY

$? 0175$

IIIPLIT PU ISCTOPIC DISTRIBUTIOI AS ATOH PERCEIYTS

PU23\%

7.003

Fue39

797.617

PU 240

? 2.324

Fije4l

$? .0 .54$

PLIE4E

?. .002

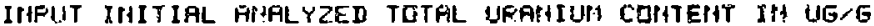

$\rightarrow 0$.

IPFUT IPITIAL AHAL'RZED AHIESICIUH EOHTEHT IH UG/G

$? 0$.

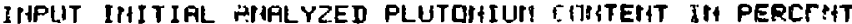

7100.

IIPUT IUUIBER DF YEARS TO BE COVEPED IH THE TABLES AS YY.

THE IECIMAL IS REQUIRED, FIID HOT HOPE THAHI 25 YEARS MAY BE USED

$? 10$.

IIFUT A 1 FOR EAIEH UUTPUT DFTIQII DESIRED. A ZERO GIVF

IHO CUTPIJT FOR THAT TABLE

ISADIYI RUAL URAHIUH ISUTOPE GROW-IH III UG

$? 1$

TOTAL URFIIIUA GRDW-IIA IH ISG

$\hat{z} 1$

TDTHL AMERICIUH GROU-III IH UG

$? 1$

FLUTCIIIUA PURITY IIA $\because$ DUE TO DECAY

$? 1$

FU ISOTOPIC DISTRIDUTIOH AS ATOP. \% FDR ALL FU ISUTOPES

$? 1$ 


\begin{tabular}{|c|c|c|c|c|c|c|c|c|c|c|c|c|}
\hline TEAP & Wall & FEB & PIAR & AFR & HAY & JUII & JUL & AUIS & SEP & DCT & IIDV & DEC \\
\hline 1976 & 0. & .0 & -0 & .1 & .1 & . I & .1 & .1 & .2 & .2 &. $\bar{\varepsilon}$ &.$\dot{\pi}$ \\
\hline 1977 & .2 & 12 & .3 & .3 &, 3 & $* 2$ & .3 & .4 & .4 & .4 & .4 & .4 \\
\hline 1979 & .5 & .5 & .5 & .5 & .5 & .6 & .6 & .6 & .5 & .6 & . $\epsilon$ & .7 \\
\hline $1 \circlearrowleft 7 \%$ & .7 & .7 & .7 & .7 &. & .8 & .2 & .8 & .8 & .9 & .5 & .9 \\
\hline 1980 & .9 & .9 & 1.0 & 1.0 & 1.0 & 1.0 & 1.0 & 1.0 & 1.1 & 1.1 & 1.1 & 1.1 \\
\hline 1981 & 1.1 & $1 . i$ & 1.2 & 1.2 & 1.2 & 1.2 & 1.2 & 1.3 & 1.3 & 1.3 & 1.3 & 1.3 \\
\hline 1982 & 1.4 & 1.4 & 1.4 & 1.4 & 1.4 & 1.5 & 1.5 & 1.5 & 1.5 & 1.5 & 1.5 & 1.6 \\
\hline 1993 & 1.6 & 1.6 & 1.6 & 1.6 & 1.7 & $1, ?$ & 1.7 & 1.7 & 1.7 & 1.7 & 1.8 & 1.8 \\
\hline 188 & 1.8 & 1.8 & 1.3 & 1.9 & 1.9 & 1.9 & 1.9 & 1.9 & 1.9 & 2.0 & 2.0 & 2.0 \\
\hline $1985 i$ & 2.0 & 2.0 & 2.1 & 2.1 & $2 . i$ & 2. 1 & 2. 1 & Z. 1 & 2.2 & 2.2 & 2.2 & 2.2 \\
\hline & 2.2 & 0. & 0. & 0. & 0. & 0 & 0 & 0. & 0. & 0 & D. & 9. \\
\hline
\end{tabular}

i) 235 GRDL-IIH IN UG,

EYAMPLE CRLCULATICN FDF 10 YERR IECAY FERIOD

\begin{tabular}{|c|c|c|c|c|c|c|c|c|c|c|c|c|}
\hline IEEFR & JAII & FEE & HaR & FiPR & $H A Y^{\prime}$ & JIJit & LUL & FUG & SEP & DCT & 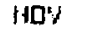 & IIEC \\
\hline $\begin{array}{l}1976 \\
1978\end{array}$ & $a_{2}$ & 2. 3 & 4.5 & 6.8 & 3.1 & 11.4 & 13.6 & $15-9$ & 13.2 & 201.5 & 22.7 & 5.0 \\
\hline 197 & & $\begin{array}{l}29.5 \\
56.8\end{array}$ & 31. & $\begin{array}{l}34.1 \\
61.4\end{array}$ & 36. & 38 & 40 & 43.2 & 45.5 & $\begin{array}{l}47.7 \\
75.0\end{array}$ & $\begin{array}{r}0.0 \\
>7.3\end{array}$ & 8.3 \\
\hline & & 84. & & & & & & & & & & \\
\hline 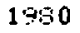 & 109 & 111.4 & 113 & 115 & 118.2 & 12 & 12 & & & & 13 & \\
\hline & & 133.6 & 14 & & & 147 & & & & 15 & & \\
\hline & & 15 & 16 & & 172.7 & & & & 181 & 18 & & \\
\hline & & & .4 & .7 & 200.0 & 20 & 204 & & & ह1 & E'1 & \\
\hline & & 4 & 222.? & & 227.3 & & & & & & & \\
\hline & & & 250.0 & .3 & 254,5 & 6.8 & & & 263 & & 68.2 & .4 \\
\hline & & & & & & & & & & & & \\
\hline
\end{tabular}


EXAMPLE CRLCULAFIUII FDR 10 YEAR IECAY PERIDD

\begin{tabular}{|c|c|c|c|c|c|c|c|c|c|c|c|c|}
\hline $\begin{array}{l}976 \\
977\end{array}$ & $\begin{array}{c}\text { JAIt } \\
0 . \\
2.4 \\
4.9 \\
7.3 \\
9.7 \\
12.2 \\
14.6 \\
17.0 \\
19.4\end{array}$ & $\begin{array}{r}\text { FEB } \\
.2 \\
2.6 \\
5.1 \\
7.5 \\
9.9 \\
12.4 \\
14.8 \\
17.2 \\
19.6 \\
22.1 \\
0.1\end{array}$ & $\begin{array}{r}\text { IAP } \\
2.4 \\
2.8 \\
5.3 \\
7.7 \\
10.1 \\
12.6 \\
1.5 .0 \\
17.4 \\
19.9 \\
22.3 \\
0.3\end{array}$ & $\begin{array}{r}\text { APR } \\
.6 \\
3.0 \\
5.5 \\
7.9 \\
10.3 \\
12.8 \\
15.2 \\
17.6 \\
20.1 \\
22.5 \\
0 .\end{array}$ & $\begin{array}{r}1 F Y \\
3 . \\
5 . \\
8 . \\
10 . \\
13 . \\
15 . \\
17 . \\
20 . \\
22 . \\
0 .\end{array}$ & $\begin{array}{c}\text { Jur1 } \\
1.0 \\
3.4 \\
5.9 \\
8.3 \\
10.7 \\
13.2 \\
15.6 \\
18.0 \\
27.0 \\
22.9 \\
0.9\end{array}$ & $\begin{array}{r}\text { JUL } \\
1.2 \\
3.6 \\
6.1 \\
8.5 \\
10.9 \\
13.4 \\
15.8 \\
18.2 \\
20.7 \\
23.1 \\
0 .\end{array}$ & $\begin{array}{r}\text { rut } \\
1.4 \\
3.9 \\
6.3 \\
3.7 \\
11.1 \\
13.6 \\
16.0 \\
18.4 \\
20.9 \\
23.3 \\
0.3\end{array}$ & $\begin{array}{r}\text { SEF } \\
1.5 \\
4.1 \\
6.5 \\
8.9 \\
11.3 \\
13.8 \\
16.8 \\
18.6 \\
21.1 \\
23.5 \\
0 .\end{array}$ & $\begin{array}{r}\text { DCT } \\
1.8 \\
4.3 \\
6.7 \\
7.1 \\
11.5 \\
14.9 \\
16.4 \\
18.8 \\
21.3\end{array}$ & $\begin{array}{r}\text { HDV } \\
2.0 \\
4.5 \\
6.9 \\
7.3 \\
11.8 \\
14.2 \\
16.6 \\
19.0 \\
21.5\end{array}$ & $\begin{array}{l}E C \\
2 .\end{array}$ \\
\hline
\end{tabular}

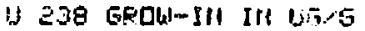

EXAIIPLE CALCULATIDIA SOR 10 YEAR DECAY PERIOD

\begin{tabular}{|c|c|c|c|c|c|c|c|c|c|c|c|c|}
\hline VEAR & JPHA & FEB & IRR & AFR & MAY & JUlt & JUL & AUG & SEP & I & $110 \psi$ & DEC \\
\hline $1 \% 76$ & 0 & 0 & .0 & .0 & . D & .0 & .0 & .0 & .0 & .0 & .0 & .0 \\
\hline 1977 & .0 & .0 & .0 & .0 & .0 & .0 & .0 & .0 & .0 & .0 & .0 & .0 \\
\hline 1978 & .0 & .0 & .0 & .0 & .0 & .0 & .0 & .0 & .0 & .0 & .0 & .0 \\
\hline 1979 & .0 & .0 & .0 & .9 & .0 & .0 & 0 & .0 & .0 & .0 & .0 & .0 \\
\hline 1930 & .0 & .0 & .6 & .0 & .0 & .0 & .0 & .0 & .0 & .0 & .0 & .0 \\
\hline $19: 31$ & 0 & .0 & .0 & .0 & .0 & .0 & .0 & .0 & .0 & 0 & .0 & .0 \\
\hline 1982 & .0 & .0 & .0 & .0 & .0 & .0 & .0 & .0 & .0 & 0 & .0 & .0 \\
\hline 1983 & . $n$ & 0 & .0 & .0 & .0 & .0 & .0 & .0 & .0 & .0 & .0 & .0 \\
\hline 1984 & .0 & .0 & .0 & .0 & .0 & .0 & . 0 & .0 & .0 & .0 & . is & .0 \\
\hline & .0 & .0 & 0 & 0 & $\therefore 0$ & .0 & .0 & 0 & .0 & .0 & $\cdot 0$ & $\therefore 0$ \\
\hline 396 & .0 & $n_{n}$ & 0. & 0. & 0 & 0. & 0. & 0. & 0. & 0. & 0. & 0. \\
\hline
\end{tabular}


TOTAL URATIUH GROW-IIt III J/5, G

EXATIPLE CFLCULATIDH FUF ta YEAP IECAY PERIDD

\begin{tabular}{|c|c|c|c|c|c|c|c|c|c|c|c|c|}
\hline $\begin{array}{l}E A P \\
976\end{array}$ & $\begin{array}{r}\text { JPHl1 } \\
0 . \\
29.9 \\
59.9 \\
89.8\end{array}$ & $\begin{array}{r}\text { FEB } \\
2.5 \\
32.4 \\
62.4 \\
92.3 \\
122.2 \\
152.1 \\
182.1 \\
212.0\end{array}$ & $\begin{array}{r}\text { IHAR } \\
5.0 \\
34.9 \\
6.4 .9 \\
94.8 \\
124.7 \\
154.6 \\
184.6 \\
214.5 \\
244.4\end{array}$ & $\begin{array}{r}\text { APR } \\
7.5 \\
37.4 \\
67.4 \\
97.3 \\
127.2 \\
157.1 \\
187.1 \\
217.0 \\
246.9\end{array}$ & $\begin{array}{r}\text { MAY } \\
10.0 \\
39.9 \\
69.8 \\
99.8 \\
129.7 \\
159.6 \\
189.5\end{array}$ & $\begin{array}{r}\text { JU11 } \\
12.5 \\
42.4 \\
72.3 \\
102.3 \\
132.2 \\
162.0 \\
192.0 \\
222.0\end{array}$ & $\begin{array}{l}\text { JUL } \\
15.0 \\
44.9 \\
74.8 \\
104.8 \\
134.7 \\
164.6 \\
194.5\end{array}$ & $\begin{array}{r}\text { AU5 } \\
17.5 \\
47.4 \\
77.2 \\
107.3 \\
137.2 \\
167.1 \\
197.0 \\
226.9 \\
256.9 \\
286.8\end{array}$ & $\begin{array}{r}\text { SEP } \\
20.0 \\
49.9 \\
79.8 \\
109.8 \\
139.7 \\
169.6 \\
199.5 \\
229.4 \\
259.4 \\
289.3\end{array}$ & $\begin{array}{r}\text { 口CT } \\
22.5 \\
52.4 \\
82.3 \\
112.2 \\
142.2 \\
172.1 \\
202.0 \\
231.9\end{array}$ & $\begin{array}{r}110 W \\
24.9 \\
54.9 \\
84.8\end{array}$ & DEC \\
\hline
\end{tabular}

TOTAL AMERICIUN GRDW-III III UG, G

EXATIPLE CALCULATIDI FOR 10 YEAR DECAY PERIUD

\begin{tabular}{|c|c|c|c|c|c|c|c|c|c|c|c|c|}
\hline $\begin{array}{l}\text { YEAR } \\
1976 \\
1977 \\
1978 \\
1979\end{array}$ & $\begin{array}{r}\text { JAll } \\
0 . \\
25.1 \\
49.0 \\
71.8 \\
93.6 \\
114.4 \\
134.2 \\
153.1 \\
171.1 \\
189.3 \\
204.7\end{array}$ & $\begin{array}{r}\text { FEE } \\
2.1 \\
27.1 \\
50.9 \\
73.7 \\
95.4 \\
116.0 \\
135.8 \\
154.6 \\
172.6 \\
189.7 \\
0 .\end{array}$ & $\begin{array}{r}\text { HAF } \\
4.3 \\
29.1 \\
52.9 \\
75.5 \\
97.1 \\
117.7 \\
137.4 \\
156.1 \\
174.0 \\
191.1 \\
0 .\end{array}$ & $\begin{array}{r}\text { APP } \\
6.4 \\
31 . \overline{5} \\
54.8 \\
77.4 \\
98.9 \\
119.4 \\
139.0 \\
157.7 \\
175.5 \\
192.5 \\
0 .\end{array}$ & $\begin{array}{r}\text { MAY } \\
8.5 \\
33.2 \\
55.7 \\
79.2 \\
100.6 \\
121.1 \\
140.6 \\
159.2 \\
176.9 \\
193.8 \\
0 .\end{array}$ & $\begin{array}{c}\text { '151t } \\
10.6 \\
35.2 \\
6.3 .0 \\
61.0 \\
102.4 \\
122.7 \\
142.2 \\
160.7 \\
178.4 \\
195.1 \\
0 .\end{array}$ & $\begin{array}{c}\text { JÜL } \\
12.7 \\
37.2 \\
00.5 \\
82.8 \\
104.1 \\
124.4 \\
143.7 \\
162.2 \\
179.8 \\
196.6 \\
0 .\end{array}$ & $\begin{array}{r}\text { AUG } \\
14.8 \\
39.2 \\
62.4 \\
84.6 \\
105.8 \\
126.0 \\
145.3 \\
163.7 \\
181.2 \\
198.0 \\
0 .\end{array}$ & $\begin{array}{r}\text { SEP } \\
16.8 \\
41.2 \\
64.3 \\
86.4 \\
107.5 \\
127.7 \\
146.9 \\
165.2 \\
182.6 \\
199.3 \\
0 .\end{array}$ & $\begin{array}{r}\text { QCT } \\
13.3 \\
43.1 \\
66.2 \\
88.2 \\
109.3 \\
129.3 \\
148.4 \\
166.7 \\
184.1 \\
200.7 \\
0 .\end{array}$ & $\begin{array}{r}\text { HOW } \\
21.0 \\
45.1 \\
68.1 \\
90.0 \\
111.0 \\
130.9 \\
150.0 \\
168.1 \\
185.5 \\
202.0 \\
0 .\end{array}$ & $\begin{array}{l}\text { DEC } \\
23.0 \\
47.0 \\
70.0 \\
91.8\end{array}$ \\
\hline
\end{tabular}




$\begin{array}{cc}\text { HOHTH } & 1976 \\ 1 & 100.0000 \\ 2 & 99.9995 \\ 3 & 99.9991 \\ 4 & 99.9986 \\ 5 & 99.9982 \\ 6 & 99.9977 \\ 7 & 99.9972 \\ 8 & 99.9968 \\ 9 & 99.9963 \\ 10 & 99.9959 \\ 11 & 99.9954 \\ 12 & 99.9950\end{array}$

$\begin{array}{cl}\text { HartTH } & 1981 \\ 1 & 99.9735 \\ 2 & 99.9732 \\ 3 & 99.9728 \\ 4 & 99.9723 \\ 5 & 99.9719 \\ 6 & 99.9715 \\ 7 & 99.9711 \\ 8 & 99.9707 \\ 9 & 99.9703 \\ 10 & 99.9699 \\ 11 & 99.9694 \\ 12 & 99.9690\end{array}$

1977
99.9945
99.9940
99.9936
99.9931
99.9927
99.9922
99.9918
99.9913
99.9909
99.9904
99.9900
99.9896

1982

99.7686

99.9692

99.9673

99.96 .4

99.9670

99.9666

99.9662

99.9658

99.9654

99.9650

99.9646

99.9641

1978
99.9891
99.9887
99.9202
99.9878
99.9873
99.9869
99.9865
99.9860
99.9856
99.9851
99.9847
99.9943

1983

99.9537

99.9633

99.3659

99. $96 \overline{5}$

99.9621

99.9617

99.9613

99.9609

99.9605

99.9601

99.9557

99.9593
1979

90.9838

99.9834

99.9830

99.9825

99.9321

99.9817

99.9812

99.9808

99.9804

99.9800

99.9795

99.9791

1954

99.9537

99.9536

99.9532

99.9578

99.9574

99.9570

99.3566

99.9562

99.9558

99.9554

99.9550

99.9546
1980

30.9787

99.9782

39.9778

39.9774

99.9770

99.9765

99.9761

99.9757

99.9753

49.9749

99.9744

99.9740

90

99.9535

99.9531

99.9527

99.9523

99.9519

99. 9515

99.9511

99.9508

99.9504

99.9500 
VAR IATIDI OF PLUTDIIIUII ISOTOPIC

EXAIPLE CALCULATIOII FUR 10 YEAR DECAY PERIDD

\begin{tabular}{|c|c|c|}
\hline ס & Y保 & $238 \mathrm{PU}$ \\
\hline Z & 70 & - 002998 \\
\hline 3 & 76 & - 002996 \\
\hline 4 & 76 & - 002994 \\
\hline 5 & 76 & .002992 \\
\hline 6 & 76 & - 002990 \\
\hline 7 & 76 & - 002988 \\
\hline 8 & 76 & - 002986 \\
\hline 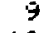 & 75 & - J0E.984 \\
\hline 10 & 75 & - 00दि:ज̆gz \\
\hline 11. & 76 & .003980 \\
\hline 12 & 76 & . 002979 \\
\hline 1 & 77 & .003977 \\
\hline 2 & 77 & .002975 \\
\hline 3 & 77 & .002973 \\
\hline 4 & 77 & . 002971 \\
\hline 5 & 77 & - 002969 \\
\hline 6 & 77 & .002357 \\
\hline 7 & 77 & .002965 \\
\hline 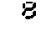 & 77 & . 002963 \\
\hline 9 & 77 & . 002961 \\
\hline 10 & 77 & .002959 \\
\hline 11 & 77 & . 002957 \\
\hline 12 & 77 & . 002955 \\
\hline 1 & 78 & .002953 \\
\hline ¿ & 78 & . 00ट95i \\
\hline 3 & 73 & .002949 \\
\hline 4 & 78 & .002949 \\
\hline 5 & 78 & - 002946 \\
\hline 6 & 78 & . 002944 \\
\hline 7 & 73 & 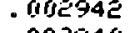 \\
\hline 8 & 73 & .002940 \\
\hline 9 & 78 & .002938 \\
\hline 10 & 78 & .002536 \\
\hline 11 & 78 & - 002934 \\
\hline 12 & 78 & - 00돌 \\
\hline 1 & 79 & .002930 \\
\hline$\hat{\varepsilon}$ & 79 & - 002928 \\
\hline 3 & 79 & . 002926 \\
\hline 4 & 79 & - 002925 \\
\hline 5 & 79 & - 00⒐3 \\
\hline 6 & 79 & - 00e9a1 \\
\hline 7 & 79 & - 002919 \\
\hline$\$$ & 79 & - 002917 \\
\hline 9 & 79 & .002915 \\
\hline 10 & 79 & .002913 \\
\hline 11 & $\begin{array}{l}79 \\
79\end{array}$ & - 002911 \\
\hline 1 & 80 & .002907 \\
\hline 2 & 80 & .002905 \\
\hline 3 & 80 & .002904 \\
\hline
\end{tabular}

239 PU
97.617223
97.617446
97.617667
97.617888
97.618108
97.618327
97.618545
97.618763
97.618980
97.619196
97.619411
97.619625
97.619839
97.620052
97.620264
97.620475
97.6201685
97.620896
97.621105
97.621313
97.621521
97.621728
97.621934
97.621139
97.622344
97.622547
97.622751
97.622953
97.623155
97.623356
97.623556
97.623755
97.623954
97.624152
97.624349
97.624546
97.624742
97.624937
97.625132
97.625325
97.625519
97.625711
97.625903
97.626094
97.626284
97.626474
97.626663
97.626851
97.627039
97.627226

$240 \mathrm{PU}$

2. 323990

2. 323981

2. 323971

2. 323961

2. 323951

2. 323941

2. 323932

2. 323922

2. 323912

2. 323902

2. 323892

2. 323882

c. 323872

E. 323862

2. 323852

2. 32.8442

2. 323832

2. 323822

2. 323812

2. 323802

2. 323792

2. 323782

2. 323772

2. 323762

2. 323752

2. 32.3742

2. 323731

2. 323721

2. 323711

2. 323701

c. 323690

2. 323680

2. 323670

2. 323660

2. 323649

2. 323639

2. 323629

2. 323618

2. 323608

2. 323597

2. 323587

2. 323576

2. 323566

2. 323556

2. 323545

2. 323535

2. 323524

2. 323514

2. 323503

2. 323492
. 053788

.053578

.053368

.053159

.052951

.052743

. 052537

.052331

.052126

.051922

.051718

.051516

.051314

.051113

.050913

.050713

.050515

.050317

.050120

.049923

. 045720

.049533

.049339

.049145

.048953

.048761

.048570

. 0483 30

. 049191

.048002

.047314

.047626

.047440

.047254

.047069

. 046835

.046701

. 046518

.045336

.046154

.045973

.045793

.045614

.045435

.045257

.045080

044903

.044727

.044552

.044378
$242 \mathrm{PU}$

. 0 rge000

- 002000

. 098000

- 002000

.002000

.002050

- 002000

- 00E0J0

.002000

.092000

.002000

, 002000

- 002000

. 902000

- v0z000

. 002000

- 002000

- Daenor

- 00ट000

.002000

- 002000

- 002000

- 0UE0 00

.002000

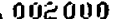

- ogeaso

002000

- jrse 000

002070

- 00ट00.1

.002000

00z000

.002000

.002000

.002000

- 0 ofizo00

.002000

.002000

.002000

.002000

.002000

002000

.002000

.002000

.002000

.002000

.002000

.002000

.002000

.002000
AT. $4 T$ T. 239.076590 239.076586 2.39. 076581 237.076 .577 239.976573 239.076569 239.076554 239.07556? 239.076556 239.076552 239.076548 239.076544 239.076539 239.076535 239. 076531 239.076527 239. 07652.3 239.076519 239. 076515 239.076511 239. 076507 239.076503 239.076499 239.076495 239.076491 239.076487 239.076483 239.075479 239.076476 239.076472 239.076468 239. 076464 239.075450 239. 075455 239.076453 239.076449 239.07644 .5 235.076441 239.075 .438 239.075434 239.076430 239.076426 239. 076423 239.076419 239. 076415 239.076412 239.076408 239.076405 239. 076401 239.076397 


\section{EXPMIPLE CALCULATIDPA FDR 10 YEAR DECAY PERIOD}

\begin{tabular}{|c|c|}
\hline 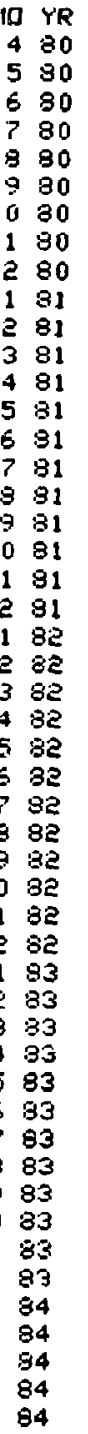 & $\begin{array}{l}238 P U \\
.002902 \\
.002900 \\
.002898 \\
.002896 \\
.002894 \\
.002892 \\
.002890 \\
.002888 \\
.002887 \\
.002885 \\
.002883 \\
.002881 \\
.002879 \\
.002877 \\
.002875 \\
.002873 \\
.002871 \\
.002870 \\
.002868 \\
.002866 \\
.002864 \\
.002862 \\
.002860 \\
.002858 \\
.002857 \\
.002855 \\
.002853 \\
.002851 \\
.002849 \\
.002847 \\
.002845 \\
.002843 \\
.002842 \\
.002840 \\
.002838 \\
.002936 \\
0002834 \\
.002832 \\
.002830 \\
.002829 \\
.002827 \\
.002825 \\
.002823 \\
.002821 \\
.002819 \\
.002818 \\
0028819 \\
.002810\end{array}$ \\
\hline
\end{tabular}

$239 \mathrm{PU}$

97.627412 97.627598

97.627783

97.627967

97.623151

97.628334

97.628516

97.628698

97.628879

97.629059

97.629239

97.629418

97.629597

97.529775

97.629952

97.630129

97.630305

97.630480

97.630655

97.630829

97.631002

97.631175

97.631347

97.631519

97.631690

97.621350

97.632030

97.632196

$97.6323+$

97.505

97.52003

97.630670

97.6351137

$97.6332 \mathrm{Je}$

97.633367

97.6 .33532

97.633696

97.633659

$97.6340 \mathrm{ez}$

97.634184

97.034346

97.634507

97.634567

97.634827

97.634986

97.635145

97.635303

97.635461

97.635618

97.635775
$240 \mathrm{FIJ}$

2. 323432

2. 323471

2. 323461

2. 323450

2. 323439

2. 323429

2. 323418

2. 323407

ㄹ. 323397

2. 323386

2. 323375

2. 323364

2. 323354

2. 323343

2. 323332

2. 323321

2. 323310

2. 323300

2. 323289

2. 323279

2. 323267

ㄱ. 323256

2. 323245

2. 323234

2. 323223

ㄷ. 323212

2. 323201

2. 323190

ㄹ. 323179

‥323168

2. 323157

2. 323!46

2. 323135

2. 223124

2. 323113

2. 323102

2. 323091

2. 353080

2. 323068

ㄱ. 323057

2. 323046

2. 323035

2. 323024

2. 323013

2. 323001

. 322990

2. 322979

2. 322968

2. 322956

2. 325945
$241 \mathrm{PU}$

- 044204

.0441131

. 043858

.043656

.043515

.043345

.043175

.043006

.042837

.042670

.042502

.042336

.042170

.042005

.041840

- 041676

.041513

.041350

.041188

.041027

.040866

040706

.040547

.040383

.040230

.040072

.039915

. 639759

039603

.039449

. 039293

.039139

. 038986

.038833

. 038621

.036530

.038379

.038228

. 038079

037929

.037781

.037633

.0 .37485

.037339

.037192

.037047

.036901

.036757

.036613

.036469
¿42 FU

- वरẼón

- vozona

- vizadoo

- ovedoo

- 0rjenta

- ocizada

- vozoua

- gré000

. 00ट001

- jo

- 0112001

- 0nenas

- oregor

- 00zocal

. 002002

- 102001

- 00z001

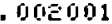

- ogzona

- joenal

- gozana

- arienrol

- ane (11) 1

- onegina

- 0uE001

- 0reona

- 00อ 001

- 00? 001

- 00zona

- पaz̃ola

- 00E001

- ogzogr

- onzonas

- 00z001

- oreona

- arjenar

- 0yE001

- 00E001

.002001

- oazaur

- ageoga

- 00z001

- 400001

- 010天01

- 002001

.002001

. 002001

.002001

002001

.002001
AT.

239.076394 Е39. 1.6390 239.076387 $2: 39.076383$ 239.075380 239.076376 239.075373 2:39. 1176.369 239. 076366 239. 076362 239.076359 23\%. 076355 239.076352 239. 076349 235.076345 239.076342 239.076 .338 239.076335 239.076332 237.1176388 239. 476325 239. 175322 239.076318 239. 076315 239. 076312 239. 076309 239. 075305 239.076352 239.076294 239.076296 239. 076293 239.076289 239.076286 239. 075 33 उ39. 076280 239. 076 239.075274 $23 \% .0762 ? 1$ 239. 076268 239.076264 239. 076261 239.076258 239.076255 239. 076 252 239.076249 239.076246 239. 076243 239.076240 239. 076237

235.076234 
EXAHPLE CALCULATIDH FOP 10 YEAR DECAY PEPIOD

$\begin{array}{rrr}110 & Y R & 238 P U \\ 6 & 84 & .002808 \\ 7 & 84 & .002807 \\ 9 & 84 & .002805 \\ 9 & 84 & .002803 \\ 10 & 84 & .002801 \\ 11 & 84 & .002799 \\ 12 & 84 & .002797 \\ 1 & 85 & .002796 \\ 2 & 85 & .002794 \\ 3 & 85 & .002792 \\ 4 & 85 & .002790 \\ 5 & 85 & .002788 \\ 6 & 85 & .002786 \\ 7 & 85 & .002795 \\ 8 & 85 & .002793 \\ 9 & 85 & .002781 \\ 10 & 85 & .002779 \\ 11 & 85 & .002777 \\ 12 & 85 & .002775 \\ 1 & 86 & .002774\end{array}$

$239 \mathrm{PIJ}$
97.635931
97.636086
97.636241
97.636395
97.636549
97.636703
97.635855
97.637008
97.637159
97.637310
97.637461
97.637611
97.637761
97.637910
97.638058
97.638206
97.638354
97.638501
97.638647
97.638793

$240 \mathrm{PU}$
2.322934
2.322922
2.322911
2.322900
2.3222888
2.322877
2.322866
2.322854
2.322843
2.322831
2.322820
2.322808
2.322797
2.322786
2.322774
2.322763
2.322751
2.322740
2.322728
2.322716

$241 \mathrm{PU}$

.036326

.036124

.036042

035901

.035761

.035620

.035481

.0 .35342

.035203

.035066

.034928

.034791

.034655

.034519

.034384

.034249

.034115

.033981

.033848

.033716
$242 \mathrm{PU}$ .002001

.002001

- 00Ẽo01

.002001

$.00 E 001$

. 00z001

- 0u2001

.002001

.002001

002001

002001

.002001

002001

.002001

.002001

.202001

002001

.002001

.002001

.002001
FT. UT. อ39.076231 239. 076228 239. 076225 ट39. 076223 239.076220 239.076217 239. 076214 239. 0762 *1 239.0762 239. 076205 239.076202 239. 1575194 239. 076197 239. 076194 239.076191 239.076188 239. 076185 239.076183 259.076180 239.076177 